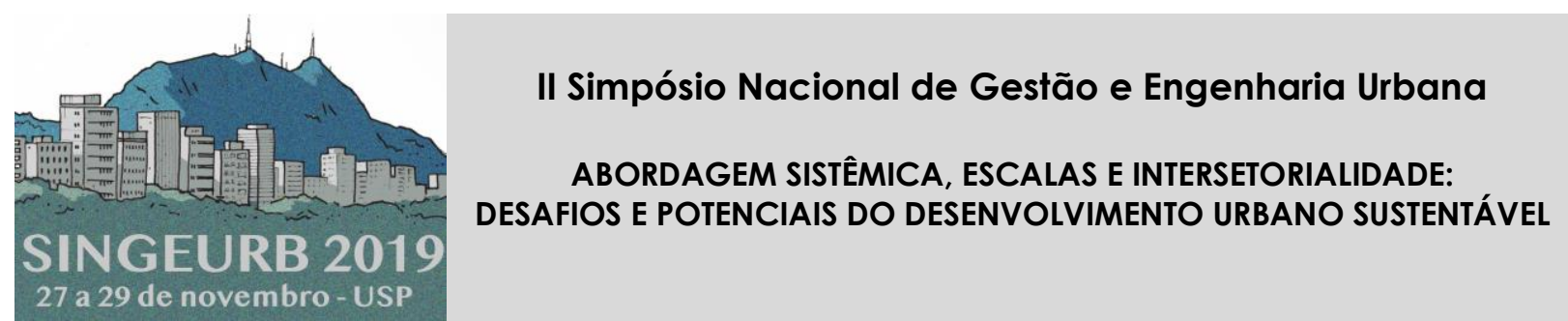

\title{
O Objetivo de Desenvolvimento Sustentável 11 nos Planos Diretores de Suzano, Mogi das Cruzes, Biritiba Mirim e Salesópolis ${ }^{1}$
}

\section{The Sustainable Development Goal 11 in the Master Plans of Suzano, Mogi das Cruzes, Biritiba Mirim and Salesópolis}

\author{
Fujimori, Maria Izabel B. 1; Abiko, Alex²; \\ 1 Escola Politécnica - USP, Av. Prof. Almeida Prado, trav.2 n. 83, Cidade \\ Universitária, São Paulo - SP, Brasil - maria.fujimori@usp.br \\ 2Escola Politécnica - USP - alex.abiko@usp.br
}

\begin{abstract}
RESUMO
Diante da necessidade de instituírem políticas públicas mais sustentáveis, os governantes precisam utilizar-se dos instrumentos municipais para garantir o desenvolvimento urbano local e regional. Os planos diretores podem auxiliar neste objetivo, já que têm o papel de estabelecerem a política de desenvolvimento urbano e buscar a melhoria da qualidade de vida da população. Este artigo analisa comparativamente planos diretores dos municípios de Suzano, Mogi das Cruzes, Biritiba Mirim e Salesópolis aos preceitos do ODS 11 da ONU. Verificouse que o apoio em escala regional entre municípios, seja de forma financeira ou técnica, não foi objeto de 3 dos 4 planos estudados. O percentual de aderência dos planos dos quatro municípios ao ODS 11 foi de aproximadamente $80 \%$. O município com plano diretor com maior aderência foi Mogi das Cruzes (95\%), e o com menor, Biritiba Mirim (70\%). Por fim, este artigo busca contribuir com governos municipais na elaboração de planos diretores mais sustentáveis e alinhados ao ODS11 da ONU.
\end{abstract}

Palavras-chave: Plano Diretor, Políticas Públicas, Objetivos de Desenvolvimento Sustentável.

\begin{abstract}
Searching for sustainable public policies, municipal authorities need instruments to guarantee local and regional urban development. Master plans can be used to achieve this goal, because they establish policies of urban development and seek to improve population's quality of life. This paper analyzes comparatively master plans of Suzano, Mogi das Cruzes, Biritiba Mirim and Salesópolis to SDG 11 of the UN. The results show that 3 of them do not mention support between cities on a regional scale. The adherence of the four Master Plans to SDG 11 is about $80 \%$. The higher adherence verified was Mogi das Cruzes (95\%) and the lower one was Biritiba Mirim (70\%). Finally, this paper seeks to contribute to municipal governments to elaborate sustainable public policies, in a regional way.
\end{abstract}

Keywords: Master Plan, Public Policies, Sustainable Development Goal.

\footnotetext{
${ }^{1}$ FUJIMORI, Maria Izabel, ABIKO, Alex. O Objetivo de Desenvolvimento Sustentável 11 nos Planos Diretores de Suzano, Mogi das Cruzes, Biritiba Mirim e Salesópolis. In: II SIMPÓSIO NACIONAL DE GESTÃO E ENGENHARIA URBANA, 28. São Paulo, 2019. São Paulo. Anais... Porto Alegre: ANTAC, 2019.
} 


\section{INTRODUÇÃO}

O desenvolvimento sustentável busca conciliar crescimento econômico com questões sociais e ambientais. Em 2015 foi elaborada a Agenda 2030 na ONU, onde foram estabelecidos os 17 Objetivos de Desenvolvimento Sustentável (PNUD, 2015).

Figura 1 - ODS

\section{(1) OBJETIV}
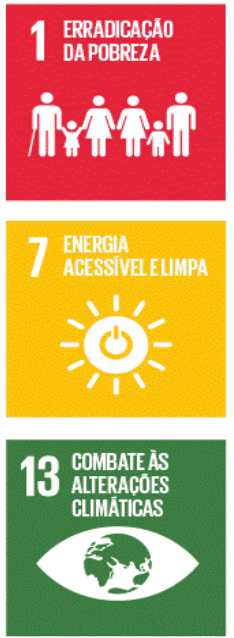
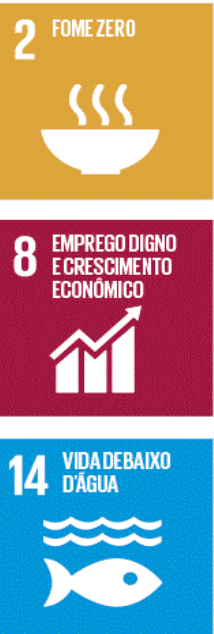
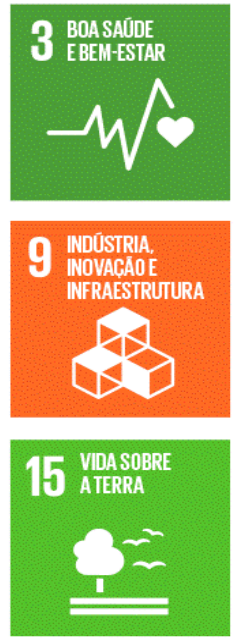

Fonte: PNUD (2015)
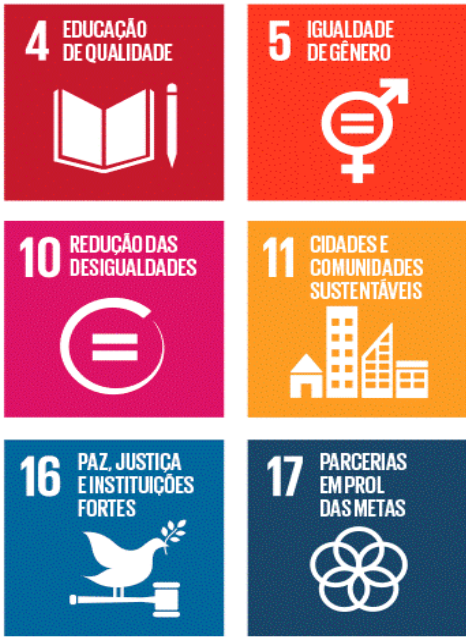

(6)

OBJETIVOS DE DESENVOLVIMENTO

Inseridas em cada um dos ODS estão metas a serem alcançadas até 2030. Buscando uma aliança entre as políticas públicas locais com as demais, estas devem identificar nos ODS bases para garantir a integração sustentável entre as esferas globais, nacionais e locais.

O ODS11 e suas metas que se referem a cidades e comunidades sustentáveis possuem implicações no planejamento municipal (BERG, 2018, p. 253). Assim, as autoridades municipais terão que mudar a forma de planejar, desenvolver, governar e gerir atrelando aspectos sustentáveis, orientados às pessoas e integrados à urbanização. (WATSON, 2016, p.439).

\subsection{0 uso de indicadores de sustentabilidade em municípios}

O papel da ONU no desenvolvimento de uma agenda sustentável estimula agentes locais a adaptarem as propostas globais em escala regional.

Nos últimos anos, sistemas de indicadores foram criados com propostas similares aos ODS. Existem ao menos 125 sistemas de indicadores e guias metodológicos ligados a cidades sustentáveis, inteligentes e resilientes (ISO, 2017). Existe ainda uma norma de indicadores de desenvolvimento sustentável, a ISO-37120/2018, cuja versão brasileira, (ABNT-NBR/ISO 37120/2017 - Desenvolvimento sustentável de comunidades - Indicadores para serviços urbanos e qualidade de vida), foi publicada de forma traduzida, e alinhada à realidade brasileira. Em 2017, alguns indicadores desta norma foram utilizados por 220 municípios paulistas no Programa Município Verde Azul (SMA, 2018).

Analisando as ações no Brasil, percebemos uma carência de dados a nível municipal, que normalmente são mitigadas por bases de dados estaduais, como a Fundação SEADE em São Paulo, ou nacionais, como o MUNIC do IBGE. O levantamento de dados a nível local ainda é escasso e está relacionado com a capacidade da prefeitura de estabelecer equipes orientadas ou a existência de secretarias interessadas no assunto. Apenas $20 \%$ dos municípios tem uma secretaria exclusiva de planejamento urbano (IBGE, 2015). 


\subsection{Planos diretores dos municípios do Alto Tietê Cabeceiras}

A Região Metropolitana de São Paulo abrange 39 municípios sendo o maior polo de riqueza nacional. Criada em 1973, foi reorganizada em 2011 pela LC no 1.139 que instituiu o Conselho de Desenvolvimento agrupando seus municípios em sub-regiões. (SÃO PAULO, 2011).

Situados na sub-região leste, os municípios de Alto Tietê Cabeceiras (figura 2) abrigam o Sistema produtor Alto Tietê, responsável pelo abastecimento de água de 4,5 milhões de habitantes. Por esse motivo, é de grande relevância a formação de políticas públicas que mitiguem impactos ambientais locais e regionais.

Figura 2 - RMSP com área dos municípios do estudo

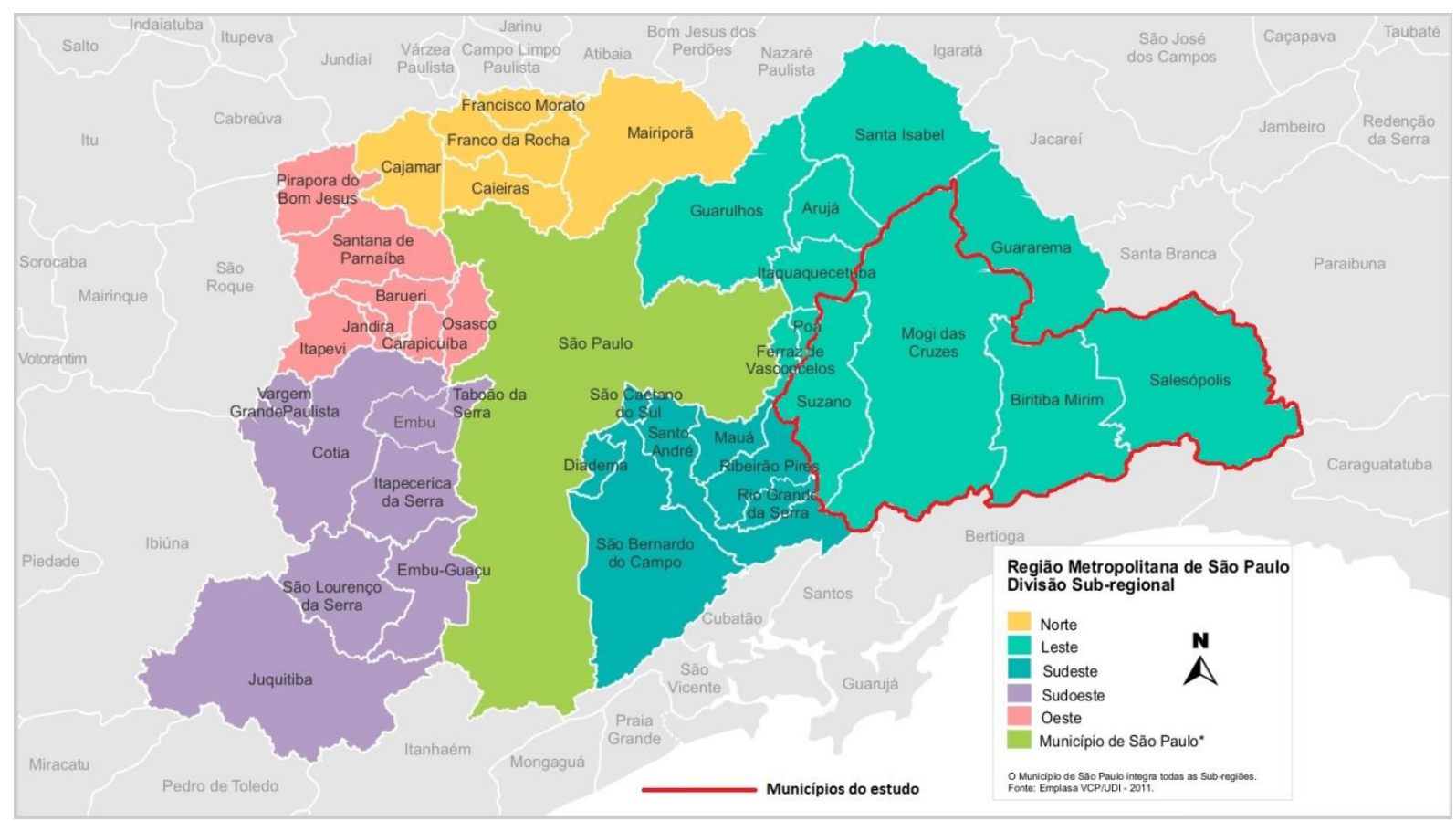

Fonte: Emplasa (2011)

Os municípios apresentados estão em fase de revisão ou recentemente revisaram seus planos diretores (quadro 1).

Quadro 1: Datas e status dos planos diretores dos municípios do estudo

\begin{tabular}{|c|c|c|c|}
\hline SUZANO & MOGI DAS CRUZES & BIRITIBA MIRIM & SALESÓPOLIS \\
\hline $22 / 12 / 2017$ & $17 / 11 / 2006$ & $26 / 11 / 2004$ & $19 / 04 / 2018$ \\
\hline
\end{tabular}

\begin{tabular}{|c|c|}
\hline & LEGENDA \\
\hline & Em revisão \\
\hline & Revisado \\
\hline
\end{tabular}

Fonte: Planos Diretores dos Municípios²

\section{OBJETIVO E METODOLOGIA}

Este artigo busca analisar comparativamente planos diretores dos municípios de Suzano, Mogi das Cruzes, Biritiba Mirim e Salesópolis aos preceitos do ODS1 1 da ONU.

Uma planilha resumo foi produzida para visualizar o nível de aderência de ODS aos planos

${ }^{2}$ SUZANO(2017), MOGI DAS CRUZES(2006), BIRITIBA MIRIM(2004) e SALESÓPOLIS(2018). 
diretores. O processo de análise foi descrito indicando os pontos citados em cada plano, indicadores e interpretações adotadas na comparação.

Através de uma metodologia comparativa, o conteúdo dos planos diretores foi confrontado com as metas do ODS 1 1. Algumas adaptações escalares foram realizadas: em situações em que o ODS é tratado de forma global, foi transposto para a escala municipal. As relações foram qualitativamente classificadas em direta, indireta e nula.

Entende-se como "relação direta" a citação de todos os aspectos daquela meta do ODS no plano diretor. A "relação indireta" se dá quando no texto, ou pela interpretação dele, não houver menção de mesmas metas e indicadores, porém os programas do plano podem contribuir indiretamente para a meta da ODS. Por fim, quando não houver menção do assunto da meta da ODS ou o próprio texto do plano diretor tiver uma característica local não relacionada ao contexto do ODS, considera-se como "não tem relação".

Pontuaram-se as relações diretas com valor "1", as relações indiretas com valor "0,5" e a falta de relação, com "0". Assim foi possível avaliar comparativamente o grau de aderência dos municípios como um todo e individualmente ao ODS1 1.

\section{ANÁLISE E DISCUSSÃO DOS RESULTADOS}

No quadro 2 são apresentadas as relações de cada plano diretor ao ODS1 1. As adaptações de escala das metas do ODS de global para municipal estão em vermelho. 
Quadro 2 - Resultados da aderência dos PD ao ODS 11

\begin{tabular}{|c|c|c|c|c|c|}
\hline & OBJETIVO & \multicolumn{4}{|c|}{ MUNICÍPIOS } \\
\hline 11 & $\begin{array}{c}\text { ODS11: Tornar as cidades e os assentamentos } \\
\text { humanos inclusivos, seguros, resilientes e } \\
\text { sustentáveis }\end{array}$ & SUZANO & $\begin{array}{l}\text { MOGI DAS } \\
\text { CRUZES }\end{array}$ & $\begin{array}{l}\text { BIRITIBA } \\
\text { MIRIM }\end{array}$ & SALESÓPOLIS \\
\hline 11.1 & $\begin{array}{c}\text { garantir o acesso de todos à habitação segura, } \\
\text { adequada e a preço acessível, e aos serviços } \\
\text { básicos e urbanizar as favelas }\end{array}$ & & & & \\
\hline 11.2 & $\begin{array}{c}\text { proporcionar o acesso a sistemas de transporte } \\
\text { seguros, acessíveis, sustentáveis e a preço } \\
\text { acessível para todos, melhorando a segurança } \\
\text { rodoviária por meio da expansão dos transportes } \\
\text { públicos, com especial atenção para as } \\
\text { necessidades das pessoas em situação de } \\
\text { vulnerabilidade, mulheres, crianças, pessoas com } \\
\text { deficiência e idosos }\end{array}$ & & & & \\
\hline 11.3 & $\begin{array}{l}\text { aumentar a urbanização inclusiva e sustentável, e } \\
\text { as capacidades para o planejamento e gestão } \\
\text { de assentamentos humanos participativos, } \\
\text { integrados e sustentáveis, em todos os municípios }\end{array}$ & & & & \\
\hline 11.4 & $\begin{array}{c}\text { fortalecer esforços para proteger e salvaguardar } \\
\text { o patrimônio cultural e natural do mundo }\end{array}$ & & & & \\
\hline 11.5 & $\begin{array}{c}\text { reduzir significativamente o número de mortes e o } \\
\text { número de pessoas afetadas por catástrofes e } \\
\text { substancialmente diminuir as perdas econômicas } \\
\text { diretas causadas por elas em relação ao produto } \\
\text { interno bruto global, incluindo os desastres } \\
\text { relacionados à água, com o foco em proteger os } \\
\text { pobres e as pessoas em situação de } \\
\text { vulnerabilidade }\end{array}$ & & & & \\
\hline 11.6 & $\begin{array}{c}\text { reduzir o impacto ambiental negativo per capita } \\
\text { das cidades, inclusive prestando especial } \\
\text { atenção à qualidade do ar, gestão de resíduos } \\
\text { municipais e outros }\end{array}$ & & & & \\
\hline 11.7 & $\begin{array}{l}\text { proporcionar o acesso universal a espaços } \\
\text { públicos seguros, inclusivos, acessíveis e verdes, } \\
\text { particularmente para as mulheres e crianças, } \\
\text { pessoas idosas e pessoas com deficiência }\end{array}$ & & & & \\
\hline $11 . a$ & $\begin{array}{c}\text { apoiar relações econômicas, sociais e ambientais } \\
\text { positivas entre áreas urbanas, periurbanas e rurais, } \\
\text { reforçando o planejamento nacional e regional } \\
\text { de desenvolvimento }\end{array}$ & & & & \\
\hline $11 . b$ & $\begin{array}{c}\text { aumentar substancialmente o número de cidades } \\
\text { e assentamentos humanos adotando e } \\
\text { implementando políticas e planos integrados } \\
\text { para a inclusão, a eficiência dos recursos, } \\
\text { mitigação e adaptação às mudanças climáticas, } \\
\text { a resiliência a desastres; e desenvolver e } \\
\text { implementar, de acordo com o Marco de Sendai } \\
\text { para a Redução do Risco de Desastres 2015-2030, } \\
\text { o gerenciamento holístico do risco de desastres } \\
\text { em todos os níveis }\end{array}$ & & & & \\
\hline $11 . c$ & $\begin{array}{l}\text { apoiar os municípios menos desenvolvidos, } \\
\text { inclusive por meio de assistência técnica e } \\
\text { financeira, para construções sustentáveis e } \\
\text { resilientes, utilizando materiais locais }\end{array}$ & & & & \\
\hline & & & & & KELAÇÃO \\
\hline & & & & DIRETA & \\
\hline & & & & INDIRETA & \\
\hline & & & & NÃO TEM & \\
\hline
\end{tabular}

Fonte: Os autores

Através da pontuação realizada, pôde-se quantificar a aderência dos planos diretores ao 
ODS e analisar a matriz de forma vertical (por município), ou horizontal (por subitem do ODS em relação aos quatro municípios).

Tabela 1 - aderência ao ODS1 1 por município

\begin{tabular}{cccc}
\hline SUZANO & $\begin{array}{c}\text { MOGI DAS } \\
\text { CRUZES }\end{array}$ & $\begin{array}{c}\text { BIRITIBA } \\
\text { MIRIM }\end{array}$ & SALESÓPOLIS \\
\hline $75 \%$ & $95 \%$ & $70 \%$ & $75 \%$ \\
& Fonte: Os autores &
\end{tabular}

Tabela 2 - aderência regional dos quatro municípios a cada subitem do ODS1 1

\begin{tabular}{lll}
\hline SUB & DESCRIÇÃO & PONTOS MÁXIMO RELAÇÃO \\
ITEM
\end{tabular}

$\begin{array}{lllll}11.1 & \text { garantir o acesso de todos à habitação segura, adequada e a } & 3,5 & 4 & 88 \%\end{array}$ preço acessível, e aos serviços básicos e urbanizar as favelas

11.2 proporcionar o acesso a sistemas de transporte seguros, acessíveis, sustentáveis e a preço acessível para todos, melhorando a segurança rodoviária por meio da expansão dos transportes públicos, com especial atenção para as necessidades das pessoas em situação de vulnerabilidade, mulheres, crianças, pessoas com deficiência e idosos

11.3 aumentar a urbanização inclusiva e sustentável, e as capacidades para o planejamento e gestão de assentamentos humanos participativos, integrados e sustentáveis, em todos os municípios

\begin{tabular}{|l|l|c|c|c|}
\hline 11.4 & $\begin{array}{l}\text { fortalecer esforços para proteger e salvaguardar o patrimônio } \\
\text { cultural e natural do mundo }\end{array}$ & 4 & 4 & $100 \%$ \\
\hline 11.5 & reduzir significativamente o número de mortes e o número de & 2,5 & 4
\end{tabular}
pessoas afetadas por catástrofes e substancialmente diminuir as perdas econômicas diretas causadas por elas em relação ao produto interno bruto global, incluindo os desastres relacionados à água, com o foco em proteger os pobres e as pessoas em situação de vulnerabilidade

\begin{tabular}{|l|l|c|c|c|}
\hline 11.6 & $\begin{array}{l}\text { reduzir o impacto ambiental negativo per capita das cidades, } \\
\text { inclusive prestando especial atenção à qualidade do ar, } \\
\text { gestão de resíduos municipais e outros }\end{array}$ & 3,5 & 4 & $88 \%$ \\
\hline 11.7 & $\begin{array}{l}\text { proporcionar o acesso universal a espaços públicos seguros, } \\
\text { inclusivos, acessíveis e verdes, particularmente para as mulheres } \\
\text { e crianças, pessoas idosas e pessoas com deficiência }\end{array}$ & 3 & 4 \\
\hline
\end{tabular}

\begin{tabular}{|c|l|c|c|c|}
\hline $11 . a$ & $\begin{array}{l}\text { apoiar relações econômicas, sociais e ambientais positivas } \\
\text { entre áreas urbanas, periurbanas e rurais, reforçando o } \\
\text { planejamento nacional e regional de desenvolvimento }\end{array}$ & 3,5 & 4 & $88 \%$ \\
\hline $11 . b$ & $\begin{array}{l}\text { aumentar substancialmente o número de cidades e } \\
\text { assentamentos humanos adotando e implementando políticas } \\
\text { e planos integrados para a inclusão, a eficiência dos recursos, } \\
\text { mitigação e adaptação às mudanças climáticas, a resiliência } \\
\text { a desastres; e desenvolver e implementar, de acordo com o } \\
\text { Marco de Sendai para a Redução do Risco de Desastres 2015- }\end{array}$ \\
\hline
\end{tabular}


2030, o gerenciamento holístico do risco de desastres em todos

os níveis.

\begin{tabular}{|c|l|c|c|c|}
\hline $11 . c$ & $\begin{array}{l}\text { apoiar os municípios menos desenvolvidos, inclusive por meio } \\
\text { de assistência técnica e financeira, para construções } \\
\text { sustentáveis e resilientes, utilizando materiais locais }\end{array}$ & 0,5 & 4 & $13 \%$ \\
TOTAL & 31,5 & 40 & $79 \%$ \\
\hline
\end{tabular}

Fonte: Os autores

\section{CONCLUSÕES}

Partindo do fato de que os municípios em análise têm importância na RMSP no que tange a proteção de mananciais para abastecimento, deveríamos verificar uma aderência maior ou mesmo uma preocupação especial com os temas de sustentabilidade. Assim, este artigo comparou a aderência dos quatro planos diretores do Alto Tietê Cabeceiras ao ODS11.

Verificou-se através da metodologia utilizada maior aderência do Plano Diretor de Mogi das Cruzes (95\%), resultado de um município com uma estrutura técnica mais robusta. Pelo mesmo motivo, a menor aderência verificada foi do Plano Diretor de Biritiba Mirim (70\%), cidade esta que possui mais de $85 \%$ do seu território protegido pela lei de mananciais, sendo necessário um plano mais elaborado no que se refere à sustentabilidade. Diante da necessidade da atualização do plano deste município, visto que o último foi revisado em 2004, este aspecto pode ser melhor analisado para a revisão.

De modo geral, foi observado que em todos os municípios, mais de $50 \%$ do objetivo está presente nos planos diretores, mostrando um alinhamento macro das políticas públicas ao ODS.

Ficou clara a falta de relação de todos os planos com o subitem 11.c, no que se refere ao apoio entre municípios, demonstrando um olhar voltado ao perímetro municipal, e não ao regional.

Os planos diretores são instrumentos auxiliares aos governantes em busca de cidades mais organizadas e sustentáveis, mas para atingir completamente o ODS11, fatores complementares na gestão das cidades são necessários, uma vez que esta ocorre de forma diversificada.

\section{REFERÊNCIAS}

BERG, A. V. D. The Pursuit of SDG 11 through the Lens of Integrated Development Planning. In: AUST, H. P; PLESSIS, A. (Ed.). The Globalisation of Urban Governance: Legal Perspectives of Sustainable Development Goal 11. London: Routledge, 2018. p. 250-262.

BIRITIBA MIRIM (Município) - Lei Complementar $n .^{\circ} 05$, de 26 de novembro de 2004 - Aprova o Plano Diretor do Município de Biritiba Mirim, e dá outras providências. Biritiba Mirim, SP. 27 nov. 2004. - Disponível em: <http://cmbiritibamirim.ddns.net:8081/cmbiritibamirim/ websis/siapegov/legislativo/leis/resulta_leis.php> - acesso em 14 jan. 2019.

EMPLASA Empresa Paulista de Planejamento Metropolitano S.A. - Região Metropolitana de São Paulo. Disponível em: <https://emplasa.sp.gov.br/RMSP> - acesso em 01 ago. 2019.

IBGE - Instituto Brasileiro de Geografia e Estatística - Perfil dos municípios brasileiros 2015 Planejamento Urbano. Disponível em: <https://ww2.ibge.gov.br/home/estatistica/econo mia/perfilmunic/2015/> acesso em : 05 out. 2018. 
ISO - INTERNATIONAL ORGANIZATION FOR STANDARDIZATION. ISO/TR 37121 Sustainable development in communities -- Inventory of existing guidelines and approaches on sustainable development and resilience in cities. 2017.

MOGI DAS CRUZES (Município) - Lei Complementar $n^{\circ} 46$ de 17 de novembro de 2006 Dispõe sobre o Plano Diretor do município de Mogi das Cruzes. Mogi das Cruzes, SP. 18 nov. 2006. Disponível em: http://www.mogidascruzes.sp.gov.br/pagina/secretaria-deplanejamento-e-urbanismo/plano-diretor-vigente - acesso em 05 jan. 2019.

PNUD - Programa das Nações Unidas para o Desenvolvimento - Agenda 2030. 2015. Disponível em: <http://www.agenda2030.org.br/ods/6/> - acesso em 02/02/19.

SALESÓPOLIS (Município) - Lei Complementar 009 de 19 de abril de 2018 - Dispõe sobre Plano Diretor Participativo - PDP, da Estância Turística de Salesópolis e dá outras providências. Salesópolis, SP. 20 abr. 2018. - Disponível em: <http://salesopolis.sp.gov.br/ publicacoes/Legislacao/? dir=Leis/Complementares> - acesso em 12 jan. 2019.

SÃO PAULO (Município) - Lei Complementar 1.139 de 16 de junho de 2011 - Reorganiza a Região Metropolitana da Grande São Paulo, cria o respectivo Conselho de Desenvolvimento e dá providências correlatas. Diário Oficial do Estado de São Paulo. São Paulo, SP. 17 jun. 2011.

SMA - Secretaria Estadual do Meio Ambiente - PROGRAMA MUNICÍPIO VERDE AZUL Disponível em: <https://verdeazuldigital.sp.gov.br/site/> - acesso em 14 nov. 2018.

SUZANO (Município) - LEI COMPLEMENTAR N 312 DE 22 DE DEZEMBRO DE 2017- Dispõe sobre o Plano Diretor do Município de Suzano e dá outras providências. Suzano, SP. 23 dez. 2017. Disponível em: <http://www.suzano.sp.gov.br/web/wp-content/uploads/2017/12/312-17Plano-Diretor.pdf> - acesso em 22 jan. 2019.

WATSON, V. Locating planning in the new urban Agenda of the urban sustainable development goal. Planning theory, 15(4), 2016. p.435-448. 